PROCEEDINGS OF THE

AMERICAN MATHEMATICAL SOCIETY

Volume 46, Number 3, December 1974

\title{
PIERCING POINTS ON A SPECIAL ARC
}

\section{HARVEY ROSEN}

ABSTRACT. We describe a cellular wild arc $A$ in $S^{3}$ that is the closure of the union of a null sequence of Alford arcs and show that if $S$ is an arbitrary 2-sphere containing $A$ and tame modulo $A$, then each point of $A$ is a piercing point of $S$.

In [1], Alford constructs a cellular arc $A_{0}$ in $S^{3}$ that is wild at all its points and that lies on a 2 -sphere that is tame modulo $A_{0}$. We assume familiarity with the construction of this arc from "eyebolts." However, we give here a rough description of Alford's arc. It lies on the limit of a sequence of tame spheres; those spheres in the first three stages of construction of $A_{0}$ are drawn. In the $n$th stage of construction of $A_{0}$, the $n$th sphere is obtained from the $(n-1)$ st sphere by running thirteen adjacent eyebolts around each of the larger $4(13)^{n-3}$ eyebolts (each with a slice removed) which had been attached to the $(n-2)$ nd sphere to get the $(n-1)$ st sphere. In this $n$th stage, each eyebolt, except the last one, loops the stem of the next eyebolt; the last eyebolt hangs freely. A slice is then removed from the loop of each small eyebolt to obtain a sphere. Each point of $A_{0}$ is the intersection of a nested null sequence of eyebolts. We call the endpoint $p_{0}$ of $A_{0}$ indicated in the figure the left endpoint of $A_{0}$.

Let $A_{0}, A_{1}, A_{2}, \cdots$ be a "nice" null sequence of Alford arcs in $S^{3}$ such that if (for $i=0,1,2, \ldots:$ ) $p_{i}$ denotes the left endpoint of $A_{i}$ and $q_{i}$ denotes the other endpoint of $A_{i}$, then $A_{i+1} \cap A_{i}=\left\{q_{i+1}\right\}=\left\{p_{i}\right\}$ and $A_{j} \cap$ $A_{i}=\varnothing$ for $j \neq i-1, i, i+1$. The sequence $p_{0}, p_{1}, p_{2}, \cdot: \cdot$ converges to a point $p$. Define an arc $A=\{p\} \cup\left(\bigcup_{i=0}^{\infty} A_{i}\right)$. When saying above that $A_{0}$, $A_{1}, A_{2}, \cdots$ is "nice", we mean that we can obtain $A$ another way by allowing $n-1$ extra eyebolts in the $n$th stage of Alford's construction of $A_{0}$ each to dangle freely instead of looping around the stem of the next eyebolt.

Received by the editors February 27, 1973 and, in revised form, August 28, 1973, and November 3, 1973.

AMS (MOS) subject classifications (1970). Primary 55A30, 57 A10; Secondary 54B15, 57 A35, $57 \mathrm{~A} 60$.

Key words and phrases. Two-spheres in $S^{3}$, piercing points of 2-spheres, tameness modulo an arc, cellular wild arc, Alford's arc.

Copyright $\odot$ 1974, American Mathematical Society 
For each $n$, these dangling eyebolts are located roughly $1 / 2,1 / 4, \cdots$, and $1 / 2^{n-1}$ of the distance from $p_{0}$ to $q_{0}$. The arc $A$ obtained this latter way lies on a 2 -sphere $S_{1}$ that is tame modulo $A$, and $S_{1}$ has the property that each point of $A$ is a piercing point of $S_{1}$. We now show that every other sphere $S$ which is tame modulo $A$ also satisfies such a property. The author is grateful to the referee for improvements in the proofs.

Definitions can be found in [3]. We use the following notations: $N(p, \delta)$ $=$ the $\delta$-neighborhood of $p, \mathrm{Bd}=$ boundary, Int $=$ interior, $\mathrm{Cl}=$ closure, and $\delta$ set $=a$ set whose diameter is less than $\delta$.

We need the following lemma about the above arc $A$.

Lemma 1. If $S$ is a 2-sphere in $S^{3}$ which contains $A$ and is tame modulo $A$ and if $B$ is a tame arc in $(S-A) \cup\{p\}$ with endpoint $p$, then there is a continuous function $g$ of $S^{3}$ onto itself such that $g(B)=B$ and $A=g^{-1}(p)$ is the only nondegenerate inverse set for $g$.

Proof. Since $B$ is tame in $S^{3}$, there is a tame 3 -cell $C$ containing $B$ and missing $A-\{p\}$. Since $A$ is cellular in $S^{3}$, there is a continuous function $f$ of $S^{3}$ onto itself such that $A$ is the only nondegenerate inverse set for $f\left[2\right.$, Theorem 1]. Let $Q=\operatorname{Cl}\left(S^{3}-C\right)$. Now, $f(C)$ is a 3-cell; we show that $f(Q)$ is a 3-cell, too. The 2-sphere $f(\operatorname{Bd} Q)$ is locally tame modulo $f(p)$. The arc $f(B)$ is locally tame modulo $f(p)$ and is locally peripherally unknotted at $f(p)$ by the construction of $A$. Thus $f(B)$ is tame [8, Theorem VI], and it follows from either parts (1) or (3) of [3, Theorem 8.2.4] that $f(B d Q)$ is tame. Therefore $f(Q)$ is a 3-cell.

Now there is a homeomorphism $k$ of $S^{3}$ onto itself such that $k=f^{-1}$ on $f(C)$. Then $g=k f$ is the desired function.

The next lemma easily follows from [6, Theorem 5].

Lemma 2. If $B$ and $F$ are arcs on a 2-sphere in $S^{3}$ which have a common endpoint $p$ and which are tame modulo $p$, then $B$ and $F$ are equivalently imbedded in $S^{3}$.

Theorem. If $S$ is an arbitrary 2-sphere in $S^{3}$ which contains $A$ and is tame modulo $A$, then each point of $A$ is a piercing point of $S$.

Proof. From the construction of $A$, it follows that there is a decreasing sequence $C_{1}, C_{2}, C_{3}, \cdots$ of 3 -cells whose intersection is $A$ and which has the property that for each $\epsilon_{1}>0$ there is a $\delta>0$ and an integer $N$ such that for all $i>N, N(p, \delta) \cap \operatorname{Bd}\left(C_{i}\right)$ is contained in an $\epsilon_{1} / 2$-disk in $\operatorname{Bd}\left(C_{i}\right)$. Let $C$ be the closure of either component of $S^{3}-S$. Since $A$ is cellular in $S^{3}$, 
it follows from [4, Theorem 1] that $\operatorname{Int}(C)$ is an open 3-cell.

To show that the endpoint $p$ of $A$ is a piercing point of $S$, it suffices to show that $\operatorname{Int}(C)$ is $1-L C$ in $C-\{p\}$ at $p$ [9, Theorem 1]. Let $\epsilon>0$ be so small that the other endpoint $q$ of $A$ is not in $N(p, \epsilon)$. Choose $\epsilon_{1}$ such that $0<\epsilon_{1}<\epsilon / 2$, and let $\delta$ and $N$ be as given by the property in the previous paragraph such that we al so have $\delta<\epsilon_{1} / 2$ and $S \cap N\left(p, \epsilon_{1}\right)$ is contained in an $\epsilon / 2$-disk in $S$.

Let $f$ be a continuous function from the boundary of a disk $E$ onto a subset $J$ of $N(p, \delta) \cap \operatorname{Int}(C)$. Since $N(p, \delta)-\{p\}$ is simply connected, $f$ can be extended to a continuous function $f^{*}$ of $E$ onto a set $D$ in $N(p, \delta)-\{p\}$. Choose an $i>N$ such that $J \cap C_{i}=\varnothing$. Let $K$ be the component of $D-$ $\operatorname{Bd}\left(C_{i}\right)$ that contains $J$. Therefore $K C S^{3}-C_{i}$. Since $D \cap \operatorname{Bd}\left(C_{i}\right)$ is a subset of $N(p, \delta) \cap \operatorname{Bd}\left(C_{i}\right)$, it is contained in an $\epsilon_{1} / 2$-disk $G$ in $\operatorname{Bd}\left(C_{i}\right)$. By the Tietze extension theorem, there is a continuous function $g: D \rightarrow K \cup G$ such that $g(D-K) \subset G$ and $g$ is the identity on $\mathrm{Cl}(K)$. (Cf. [3, Theorem 4.2.2].)

Since $S \cap g(D)$ is a subset of $S \cap N\left(p, \epsilon_{1}\right)$, it is contained in an $\epsilon / 2$-disk $H$ in $S$ with $p \in \operatorname{Int}(H)$. $A$ misses $S \cap g(D)$ because $S \cap g(D) \subset \mathrm{Cl}\left(S^{3}-C_{i}\right)$. Let $r$ be the first point of $A$ (as one goes from $p$ to $q$ ) that belongs to $\operatorname{Bd}(H)$, and let $p r$ be the subarc of $A$ with endpoints $p$ and $r$. Since $S \cap g(D) C$ $H-p r$, there is a subdisk $H_{1}$ of $H$ that contains $S \cap g(D)$ but not $p$.

Let $L$ be the component of $g(D)-S$ that contains $J$. Therefore $L \subset$ Int $(C)$. By the Tietze extension theorem, there is a continuous function $b$ : $g(D) \rightarrow L \cup H_{1}$ such that $h(g(D)-L) \subset H_{1}$ and $h$ is the identity on $\mathrm{Cl}(L)$. Since $h g f^{*}: E \rightarrow N(p, \epsilon) \cap(C-\{p\})$ is a continuous extension of $f, \operatorname{Int}(C)$ is $1-L C$ in $C-\{p\}$ at $p$. This shows $p$ is a piercing point of $S$.

Now let $y$ be an arbitrary point of $A$ different from $p$. We show that $y$ is a piercing point of $S$. Suppose otherwise. Let $\beta$ be a homeomorphism of $S^{3}$ onto itself such that $C$ and $\beta(C)$ are disjoint, and for each set or point $X$ in $C$, let $X^{\prime}=\beta(X)$. Let $h: S \rightarrow S^{\prime}$ be a homeomorphism such that $h(q)=$ $p^{\prime}$ and $h(A) \cap A^{\prime}=\left\{p^{\prime}\right\}$, and let $C \cup_{b} C^{\prime}$ be the space obtained by identifying $x$ with $h(x)$. Let $\pi: C \cup C^{\prime} \rightarrow C \cup_{h} C^{\prime}$ be the usual projection map.

Since $p$ is a piercing point of $S$, there is in $S^{3}$ a tame arc on $S$ with endpoint $p$ [7, Theorem 6]. Let $B$ be an arc in $(S-A) \cup\{p\}$ which is tame modulo its endpoint $p$. By Lemma 2, $B$ is tame; and by Lemma 1 , there is a continuous function $g$ of $S^{3}$ onto itself such that $g(B)=B$ and $A=g^{-1}(p)$ is the only nondegenerate inverse set for $g$. Since $g(S)$ is a 2 -sphere that is tame modulo the tame arc $g(B), g(S)$ is tame [6, Theorem 2]. Therefore $g(C)$ is a 3-cell. : But $g(C)$ and $C / A$, the decomposition space obtained by 
collapsing $A$ to a point, are the same, and it follows that $C / A$ is a 3-cell. If $L$ denotes the arc $h^{-1} \beta(A)$, a similar argument shows that $C /(A \cup L)$ $(=C / A / L)$ is a 3-cell. Then $\left(C \cup_{h} C^{\prime}\right) / \pi(A \cup L)$ is $S^{3}$ because it can be obtained by sewing together the 3-cells $C /(A \cup L)$ and $C^{\prime} /\left(h(A) \cup A^{\prime}\right)$. Since $C \cup_{h} C^{\prime}$ is $S^{3}$ [5, Theorem 5.7], it follows that the arc $\pi(A \cup L)$ is cellular. : However, the two nonpiercing points $\pi(y)$ and $\pi\left(y^{\prime}\right)$ of $\pi(S)$ cannot lie in the same cellular set [10, Theorem 2]. Thus $y$ is a piercing point of $S$ and the result follows.

Remark. Two 2-spheres containing $A$ and tame modulo $A$ need not be equivalently embedded in $s^{3}$.

Questions. Will the theorem be true if we replace $A$ by Alford's arc $A_{0}$ ? If two 2-spheres contain $A_{0}$ and are tame modulo $A_{0}$, then are the spheres equivalently embedded in $S^{3}$ ?

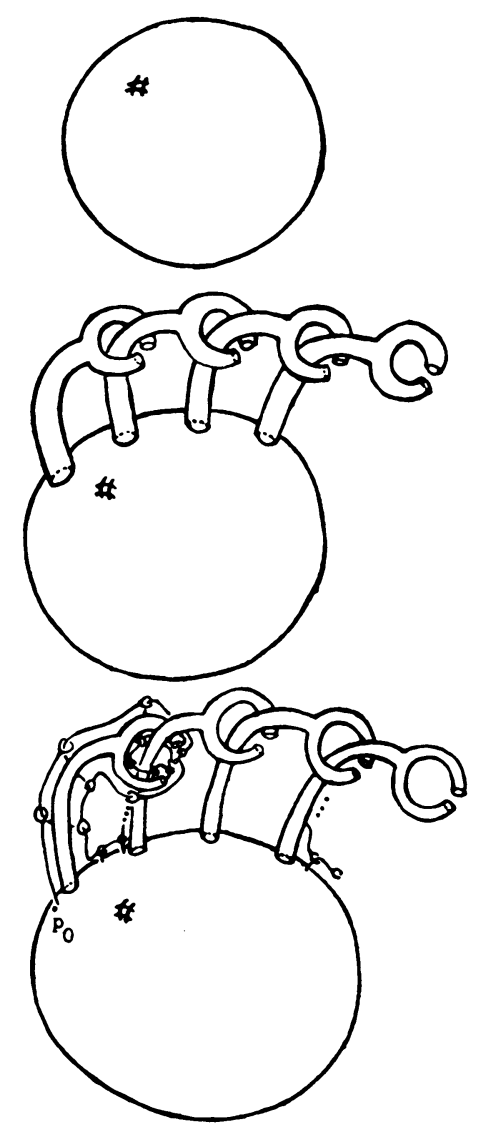




\section{HARVEY ROSEN}

\section{REFERENCES}

1. W. R. Alford, Some "nice" wild 2-spheres in E3, Topology of 3-Manifolds and Related Topics (Proc. Univ. of Georgia Inst., 1961), Prentice-Hall, Englewood Cliffs, N. J., 1962, pp. 29-33. MR 25 \#4504.

2. Morton Brown, A proof of the generalized Schoenflies theorem, Bull. Amer. Math. Soc. 66 (1960), 74-76. MR 22 \#84706.

3. C. E. Burgess and J. W. Cannon, Embeddings of surfaces in $E^{3}$, Rocky Mountain J. Math. 1 (1971), no. 2, 259-344. MR 43 \#4008.

4. J. C. Cantrell, Almost locally polyhedral 2-spheres in $S^{3}$, Duke Math. J. 30 (1963), 249-252. MR 26 \#551.

5. R. J. Daverman and W. T. Eaton, Universal crumpled cubes, Topology 11 (1972), 223-235.

6. P. H. Doyle and J. G. Hocking, Some results on tame disks and spheres in E3, Proc. Amer. Math. Soc. 11 (1960), 832-836. MR 23 \# A4133.

7. D. S. Gillman, Side approximation, missing an arc, Amer. J. Math. 85 (1963), 459-476. MR 28 \#3407.

8. O. G. Harrold, Jr., H. C. Griffith and E. E. Posey, A characterization of tame curves in three-space, Trans. Amer. Math. Soc. 79 (1955), 12-34. MR 19, 972.

9. D. R. McMillan, Jr., Some topological properties of piercing points, Pacific J. Math. 22 (1967), 313-322. MR 35 \#7319.

10. - Piercing a disk along a cellular set, Proc. Amer. Math. Soc. 19 (1968), 153-157. MR 36 \#3332.

DEPARTMENT OF MATHEMATICS, UNIVERSITY OF ALABAMA, UNIVERSITY, ALABAMA 35486 